Article

\title{
Unidirectional Invisibility Induced by Complex Anti-Parity-Time Symmetric Periodic Lattices
}

\author{
Hui Cao ${ }^{1,+}$, Dong Zhao ${ }^{2, \dagger}$, Ming Fang ${ }^{2,+}$, Huang Guo ${ }^{2}$, Yonghong $\mathrm{Hu}^{3, *}$, Fangmei Liu ${ }^{2, *}$, \\ Dong Zhong ${ }^{2}$ and Houhua Xiong ${ }^{3,+}$ \\ 1 School of Mathematics and Statistics, Hubei University of Science and Technology, Xianning 437100, China \\ 2 School of Electronics and Information Engineering, Hubei University of Science and Technology, \\ Xianning 437100, China \\ 3 School of Nuclear Technology and Chemical Biology, Hubei University of Science and Technology, \\ Xianning 437100, China \\ * Correspondence: huyonghong@hbust.edu.cn (Y.H.); liufangmei@hbust.edu.cn (F.L.) \\ + These authors contributed equally to this work.
}

Received: 3 August 2019; Accepted: 28 August 2019; Published: 11 September 2019

check for updates

\section{Featured Application: unidirectional invisibility, laser, highly sensitive sensor.}

\begin{abstract}
Complex anti-parity-time symmetric periodic lattices, in a wide frequency band, can act as unidirectional invisible media. The reflection from one end is suppressed while it is enhanced from the other. Furthermore, unidirectional laser points (ULPs) which correspond to the poles of reflection from one end, arise in the parameter space composed of the permittivity and angular frequency. The phase of the reflection coefficient changes sharply near the ULPs. Subsequently, large lateral shift which is proportional to the slope of phase could be induced for the reflected beam. The study may find great applications in unidirectional invisibility, unidirectional lasers and highly sensitive sensors.
\end{abstract}

Keywords: anti-parity-time symmetry; unidirectional invisibility; unidirectional laser; lateral shift; periodic lattice

\section{Introduction}

According to the theory of quantum mechanics, the observed physical parameters in experiments are real, and the corresponding Hamiltonian operator is Hermitian. When a light field interacts with the multi-level atomic systems, under-considering the spontaneous emission and ionization of atoms, dissipative terms are introduced, then the Hamiltonian operator to describe the system is non-Hermitian [1-3].

The eigenvalues of non-Hermitian Hamiltonian are usually complex containing imaginary parts. However, the non-Hamiltonian that satisfies the parity-time (PT) symmetry has a real spectrum, which extends quantum mechanics into a new research field [4-10]. PT-symmetry and non-Hermitian optics are new concepts and have become a research hotspot in recent years [11-19]. It is worth noting that the study of Hermitian and non-Hermitian properties are not limited to electronic systems, but also can be extended to the field of optics [20,21]. Although the Schrödinger equation describing a single particle is different from the Maxwell equation describing electromagnetic field in physical origin, the electromagnetic field propagation equation is similar to the Schrödinger equation in mathematical form under the slowly varying envelope approximation [22,23]. This similarity provides the possibility of studying PT symmetry and non-Hermiticity in optical systems. Therefore optics has the natural advantage of being used to explore all sorts of non-Hermitian predictions that used to be considered difficult in quantum systems [24-26]. The introduction of gain and loss in photonic crystals, as well as 
the clever design of various structures, can improve the luminous efficiency of materials and obtain fascinating nonlinear and topological properties [27-30].

The propagation constant in PT symmetric phase is a real number. Although there is gain and loss, the energy of the system is conserved. When the gain/loss ratio $g$ is much smaller than the coupling coefficient, it corresponds to the PT symmetric phase, and the PT symmetric supermodel appears in the system [21]. For the larger $g$, the PT phase will be broken. At this time, severe mode mismatch will occur in the two waveguides, and the energy coupling of the mode field is suppressed, resulting in the occurrence of asymmetric light intensity distribution [31]. In the vicinity of spontaneous PT-symmetry breaking point, the PT symmetric periodic structure medium shows unidirectional invisibility [32]. In a wide frequency range near the Bragg point, when light enters from one end, the outgoing light passes through unaffected. But when light comes in from the other end, the reflected light is enhanced. The anti-PT symmetry which arises from the charge-conjugation symmetry has also induced many fascinating phenomena, such as flat broadband light transport [33], topological bound modes [34] and chiral mode conversion [35]. The refractive index of material obeys $n(z)=n^{*}(-z)$ and $n(z)=-n^{*}(-z)$ in PT and anti-PT symmetric systems $[16,17,36]$, respectively. If expressed in terms of dielectric constant, PT and anti-PT symmetric systems both satisfy the condition $\varepsilon(z)=\varepsilon^{*}(-z)$. Further, we hypothesized whether new optical properties would be obtained when $\varepsilon(z)=-\varepsilon^{*}(-z)$. At this point, the refractive index satisfies $n(z)=-i n^{*}(-z)$, which is called complex anti-PT symmetry here. This new symmetry can be seen as a combination of anti-PT symmetry and rotation of $\pi / 2$. It further extends the scope of symmetry and may lead to more novel optical properties.

In this work, we investigated the unidirectional invisibility in the complex anti-PT-symmetric periodic lattices. We showed that the reflection was anisotropic as light impinged upon the lattices from the left and right end. Unidirectional laser points (ULPs) which were confirmed to be substantial boundary modes arose in the parameter space composed of the permittivity and angular frequency. We further explored the phase of reflection coefficient and demonstrated the singularity of phase and large lateral shift of reflected beam around the ULPs. The study can be utilized for highly sensitive sensing.

\section{Complex Anti-Parity-Time Symmetric Lattices}

We considered a one-dimensional complex anti-PT symmetric lattice consisting of 10 periodic unit cells, embedded in a homogeneous background, such as in air, shown in Figure 1. The dielectrics $A$ and $B$ were arranged alternately, forming a periodic structure. The structure can also be noted by $(\mathrm{AB})^{N}$, where the periodic number is $N=10$. The thickness of dielectrics A and B were $L=125 \mu \mathrm{m}$. The permittivities of the dielectrics were $\varepsilon_{a}=0.1+0.01 i$ and $\varepsilon_{b}=-0.1+0.01 i$, respectively. Based on the relation $n=\varepsilon^{1 / 2}$, the corresponding refractive indices were $n_{a}=0.3166+0.0158 i$ and $n_{b}=0.0158+$ $0.3166 i$, respectively.

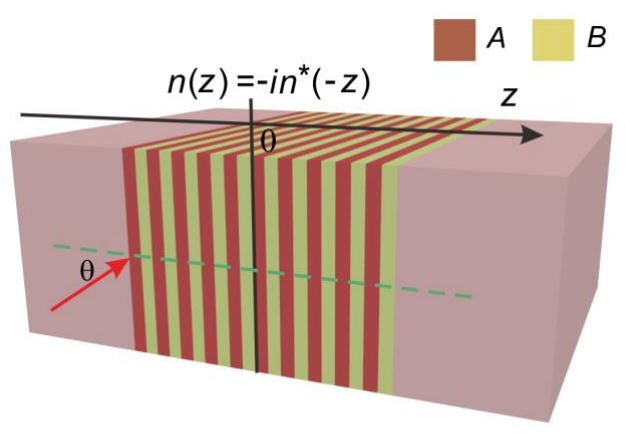

Figure 1. Schematic of the one-dimension complex anti-parity-time (PT) symmetric photonic crystals (PCs), where the complex refractive indices were distributed in $n(z)=-i n^{*}(-z)$. For the primitive unit-cell layers A and B, the thickness was $L=125 \mu \mathrm{m}$ and the complex refractive indices were $n_{a}=0.3166+0.0158 i$ and $n_{b}=0.0158+0.3166 i$, respectively. The imaginary part was positive for gain material and negative for loss material. 
Here we assumed that the input intensity of light was low enough and ignored the nonlinear effect. The materials were treated to be uniform and isotropic. As a light beam impinged upon the system, the reflection and transmission coefficients were denoted by $r$ and $t$, respectively. The two coefficients could be obtained by the transfer matrix method (TMM) $[37,38]$. The transmittance $T$ was identified as $T=t t^{*}=I_{t} / I_{i}$, where $I_{t}$ and $I_{i}$ are the transmitted and incident intensities, respectively. The reflectance $R$ was identified as $R=r r^{*}$.

\section{Unidirectional Invisibility and Laser Points}

We calculated the transmission and reflection spectra as the transverse magnetic (TM) wave obliquely impinged upon the complex anti-PT symmetric lattices. The incident angle was $\theta=5^{\circ}$. Figure $2 \mathrm{a}$ shows the transmittance and reflectance spectra for light incident from the left. The transmittance $T_{1}$ decayed rapidly and tended to a constant as the angular frequency $\omega$ increased. However, there were two peaks in the curve of reflectance $R_{1}$. The two maxima were $R_{1}=10.5$ and $7.97 \times 10^{4}$ in the curve marked by black asterisks, respectively locating at $\omega=1.83 \times 10^{13}$, and $4.29 \times 10^{13} \mathrm{rad} / \mathrm{s}$.
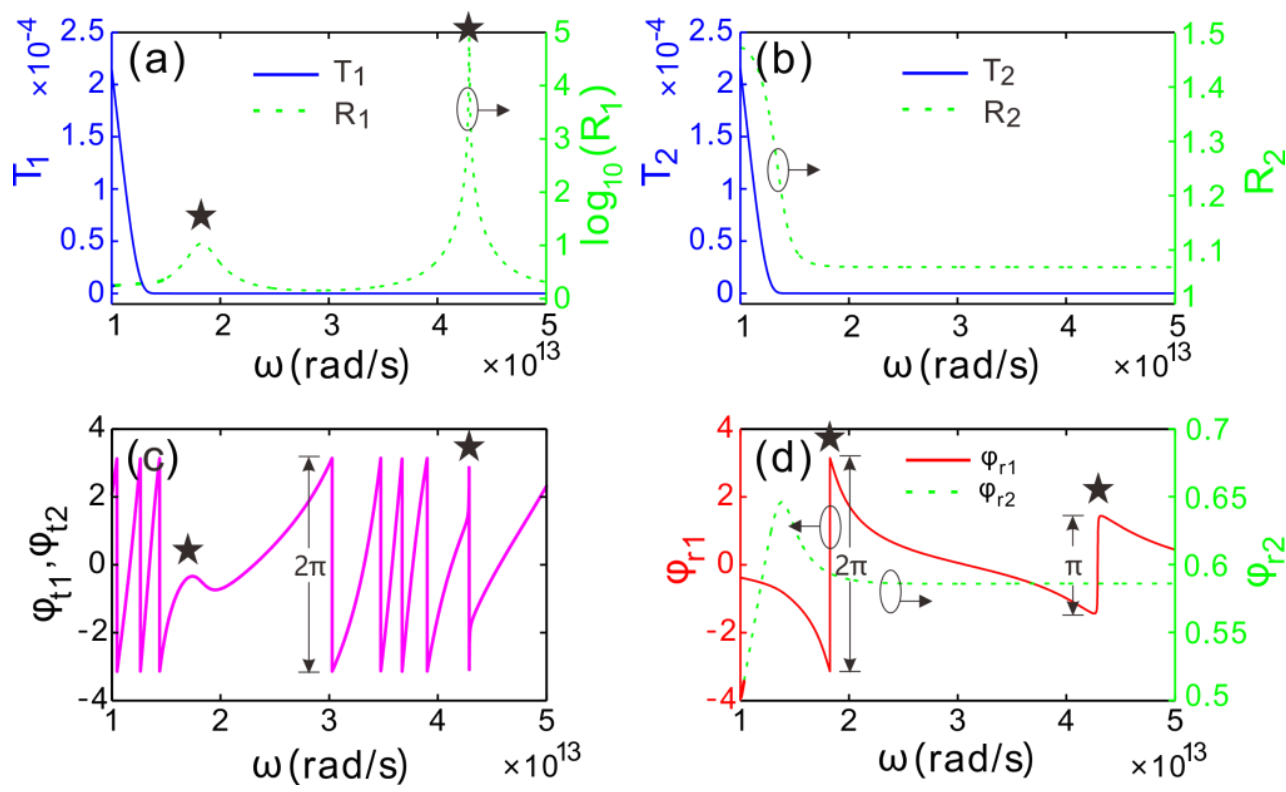

Figure 2. (a,b) The transmission and reflection spectra for light incident from the left and right, respectively. (c,d) The phases of transmission coefficient and reflection coefficient for the complex anti-PT symmetric PCs, respectively.

When light was incident from the right side, Figure $2 \mathrm{~b}$ shows the reflection spectrum and transmission spectrum. Initially, the transmittance $T_{2}$ and reflectance $R_{2}$ decreased rapidly with the increase of the angular frequency. Then, as the frequency increased, the reflectance and transmittance tended to be constant. For incident light at higher frequencies, the transmittance was close to zero. This phenomenon indicated that the media had anisotropy of the reflection as light was incident from the left and right. Therefore, for incident light with a certain frequency, the reflected light on the left could be enhanced greatly, but the reflected light on the right may be suppressed. These properties could be used to enhance the reflected light intensity from unidirection, as well as unidirectional invisibility.

The transmission and reflection coefficients were represented by $t=|t| \exp \left(i \varphi_{t}\right)$ and $r=|r| \exp \left(i \varphi_{r}\right)$, where $\varphi_{t}$ and $\varphi_{r}$ were the phase shifts of the transmitted and reflected beams referred to the incident beam, respectively. Figure $2 \mathrm{c}$ shows the phases of the transmission coefficients of light from the left and right, respectively. One can see that the reflection coefficients in the left and right were isotropic. There were many step points in the curve, and the jump value was $2 \pi$, which was meaningless and 
could be eliminated. However, near the poles of the transmittance, the phase of the transmission coefficient changed dramatically, which indicated that large lateral shift could have existed in the transmitted beam.

Figure $2 \mathrm{~d}$ shows the phase of the reflection coefficient. When light was incident from the left, the phase change of the reflection coefficient near the two maxima of reflectance was $2 \pi$ and $\pi$, respectively, and the phase varied dramatically with the angular frequency around those asterisks. The $2 \pi$ phase jump point was stepwise and the step was meaningless. But the phase changed continuously around the $\pi$ change point. When light was incident from the right, the phase curve had a maximum point. With the increase of angular frequency, the phase of reflection coefficient first increased, then decreased, and finally tended to a constant value. For the reflected beam, the change in phase was extremely sensitive to the angular frequency of incident light around the maxima of the reflectance, so this effect could be used for high-sensitivity sensors.

To find the properties in the complex anti-PT symmetric lattices, we defined the permittivities of dielectrics $\mathrm{A}$ and $\mathrm{B}$ as $\varepsilon_{a}=0.1+0.01 \varepsilon_{i} i$ and $\varepsilon_{b}=-0.1+0.01 \varepsilon_{i} i$, respectively. The gain in the lattices could be obtained by nonlinear two-wave mixing or a Ge/Cr doped fiber [39,40], while the loss could be achieved by an acoustic modulator [41]. Figure 3a gives the reflectance $R_{1}$ as light was incident from the left. The parameter space was composed of the permittivity $\varepsilon_{i}$ and angular frequency $\omega$. One could find that there were two poles in the parameter space, denoted by the unidirectional laser point $(\mathrm{ULP})_{1}\left(\varepsilon_{i 1}=2, \omega_{1}=1.8 \times 10^{13} \mathrm{rad} / \mathrm{s}\right)$ and $\mathrm{ULP}_{2}\left(\varepsilon_{i 2}=1, \omega_{2}=4.29 \times 10^{13} \mathrm{rad} / \mathrm{s}\right)$, respectively. The corresponding maxima of the reflectance were $R_{\mathrm{UPL} 1}=2.28 \times 10^{4}$ and $R_{\mathrm{UPL} 2}=4.44 \times 10^{4}$.
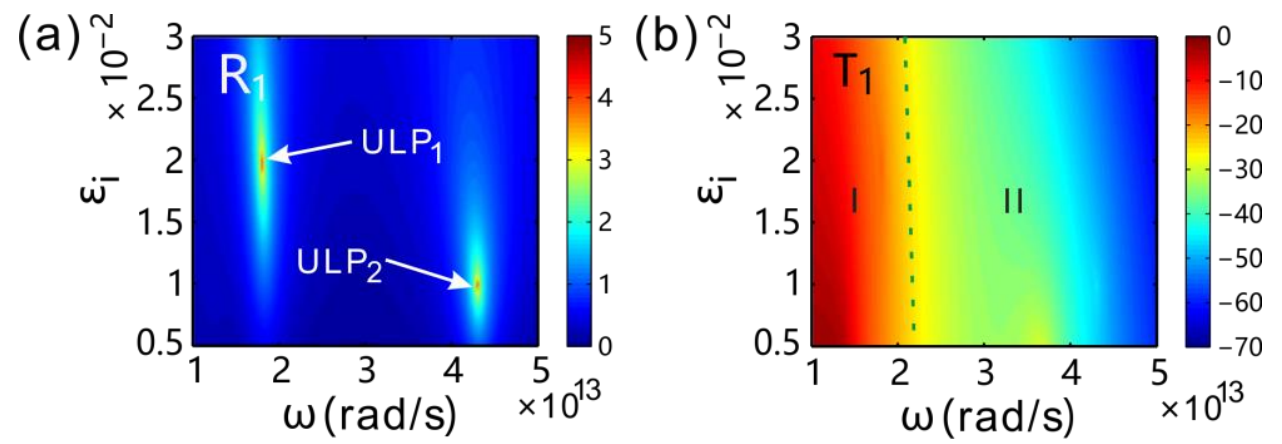

(c)

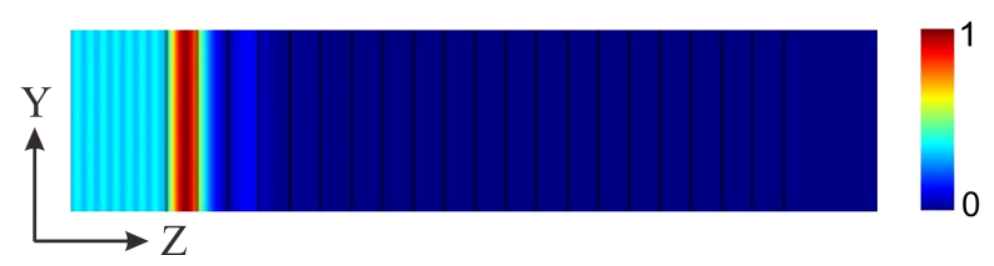

Figure 3. (a,b) The transmittance and reflectance varying with the permittivity and angular frequency for light incident from the left, respectively. (c) The electric field intensity $\left(\left|E_{\mathrm{z}}\right|^{2}\right)$ distribution at the unidirectional laser point $\left(\mathrm{ULP}_{1}\right)$.

On the contrary, we plotted the reflectance as light impinged into the lattices from the right. The intensity of reflection beam was extremely faint as shown in Figure $3 \mathrm{~b}$. The reflectance sharply decreased with the increase of the angular frequency in area I and the value of reflection in area II approached to zero in our calculating accuracy. The properties demonstrated that there was strong dependence of reflection on the two incident directions from the left and right. Especially, the poles of reflectance arose in the parameter space for the light incident from the left, but the reflectionless phenomenon could have resulted for light incident from the right. The characteristics could be therefore utilized for the unidirectional laser. Figure $3 c$ provides the distribution of the electric field intensity for the ULP ${ }_{1}$. It shows that the power of the mode field was mainly localized at the first layer of the input port, so the $\mathrm{ULP}_{1}$ could be viewed as a boundary mode. 
We studied the phase properties of reflected and transmitted coefficients as the permittivity of the dielectrics and the angular frequency changed. Figure 4 a plots the reflection phase in the parameter space. It shows the phase dislocated at the $\mathrm{ULP}_{1}$ and $\mathrm{ULP}_{2}$, which were the singular points in phase of reflected coefficient. To demonstrate the characteristics of the phase varying with the permittivity and angular frequency, Figure $4 \mathrm{~b}$ plots the phase curves for three specific permittivities $\varepsilon_{i}=0.01,0.015$ and 0.02 . One can see that the phase varied with the angular frequency. There was $2 \pi$ phase jump at the frequency $\omega_{1}$ for the curve corresponding to $\varepsilon_{i}=0.02$ and $\pi$ phase jump at the frequency $\omega_{1}$. Actually, the curve was continuous at $\omega_{1}$ since the $2 \pi$ phase jump was meaningless. For $\varepsilon_{i}=0.015$, the phase changed dramatically around the $\mathrm{ULP}_{1}$ and $\mathrm{ULP}_{2}$. This behavior indicated that large lateral shift of the reflected beam may have been induced, since the lateral shift was proportional to the curvature of the phase curve. For $\varepsilon_{i}=0.01$, the phase changed abruptly with $\pi$ at $\omega_{2}$. The $\pi$ change in phase justly approved the characteristics of the $\mathrm{ULP}_{1}$ and $\mathrm{ULP}_{2}$. The maximum property of transmittance resulted in the uncertainty in phase. We also gave the phase of transmitted coefficient in the parameter space as shown in Figure 4c. It manifested that the phase of transmitted beam also dislocated at the ULP $\mathrm{P}_{1}$ and $\mathrm{ULP}_{2}$. Figure $4 \mathrm{~d}$ demonstrates the phase changed with the angular frequency. For the above three specific permittivities, the phase abruptly stepped with $\pi$ around the $\mathrm{ULP}_{1}$ and $\mathrm{ULP}_{2}$, and at the other step points, the phase change was the no meaning value of $2 \pi$.
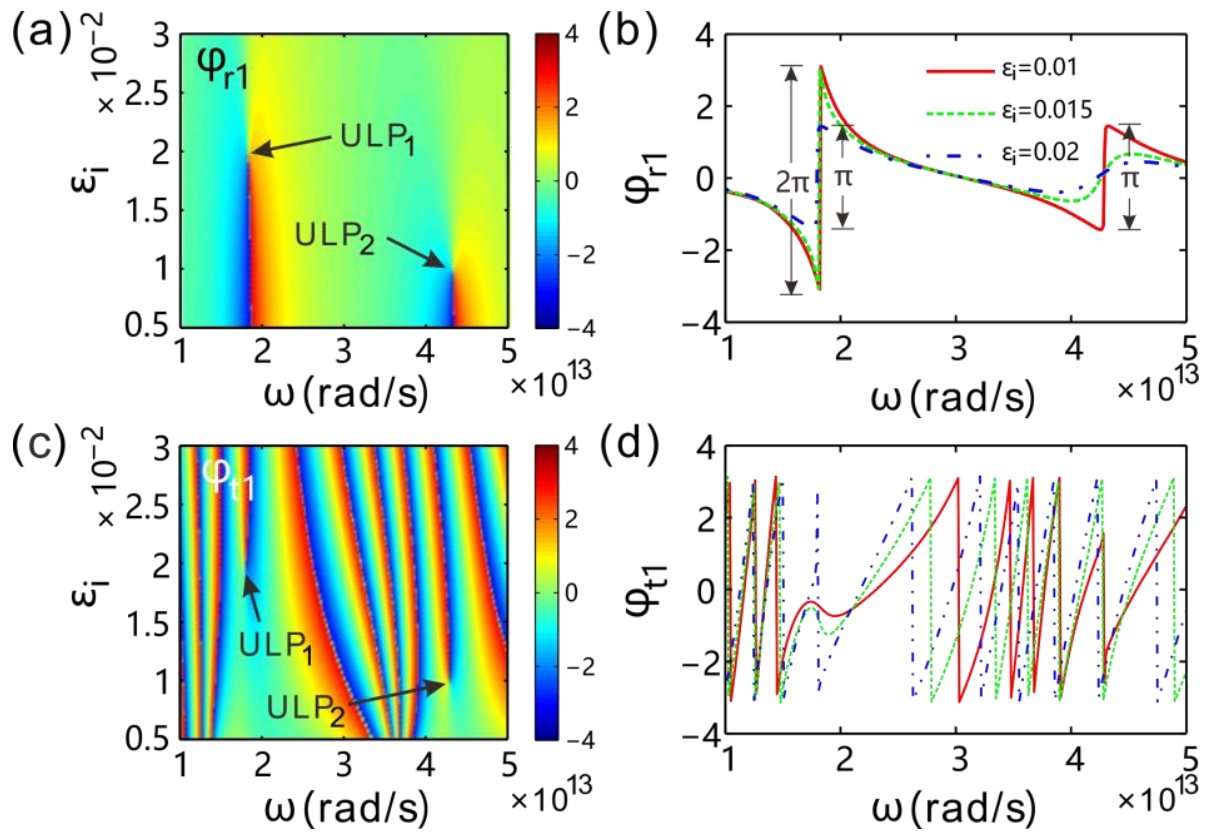

Figure 4. $(\mathrm{a}, \mathrm{c})$ The phases of reflection and transmission coefficient varying with the permittivity and angular frequency, respectively. $(\mathbf{b}, \mathbf{d})$ The phases of reflection coefficient and transmission coefficient varying with the angular frequency for three different permittivities $\varepsilon_{i}=0.01,0.015$ and 0.02 , respectively. The light was incident from the left for $(\mathbf{a}-\mathbf{d})$.

For light incident from the right, Figure 5a shows the reflectance in the parameter space. One can see the focusing area in the parameter space was divided into two parts, viz part I and part II, which corresponded to the descent zone and saturation zone, respectively. The reflectance decreased with the increase of the angular frequency in the descent zone. However, the reflectance was saturated as the angular frequency further increased. To illustrate this further, we plotted three curves of reflectance for $\varepsilon_{i}=0.01,0.015$ and 0.02 as shown in Figure 5b. In part I, the reflectance rapidly dropped as the frequency increased. In part II, the reflectance remained constant. The corresponding saturation value of reflectance was smaller for a larger permittivity. In general, the reflected beam was weak as light was incident from the right. This flaw will limit the application in practice though large lateral shift of the reflected beam may be achieved [42-44]. 

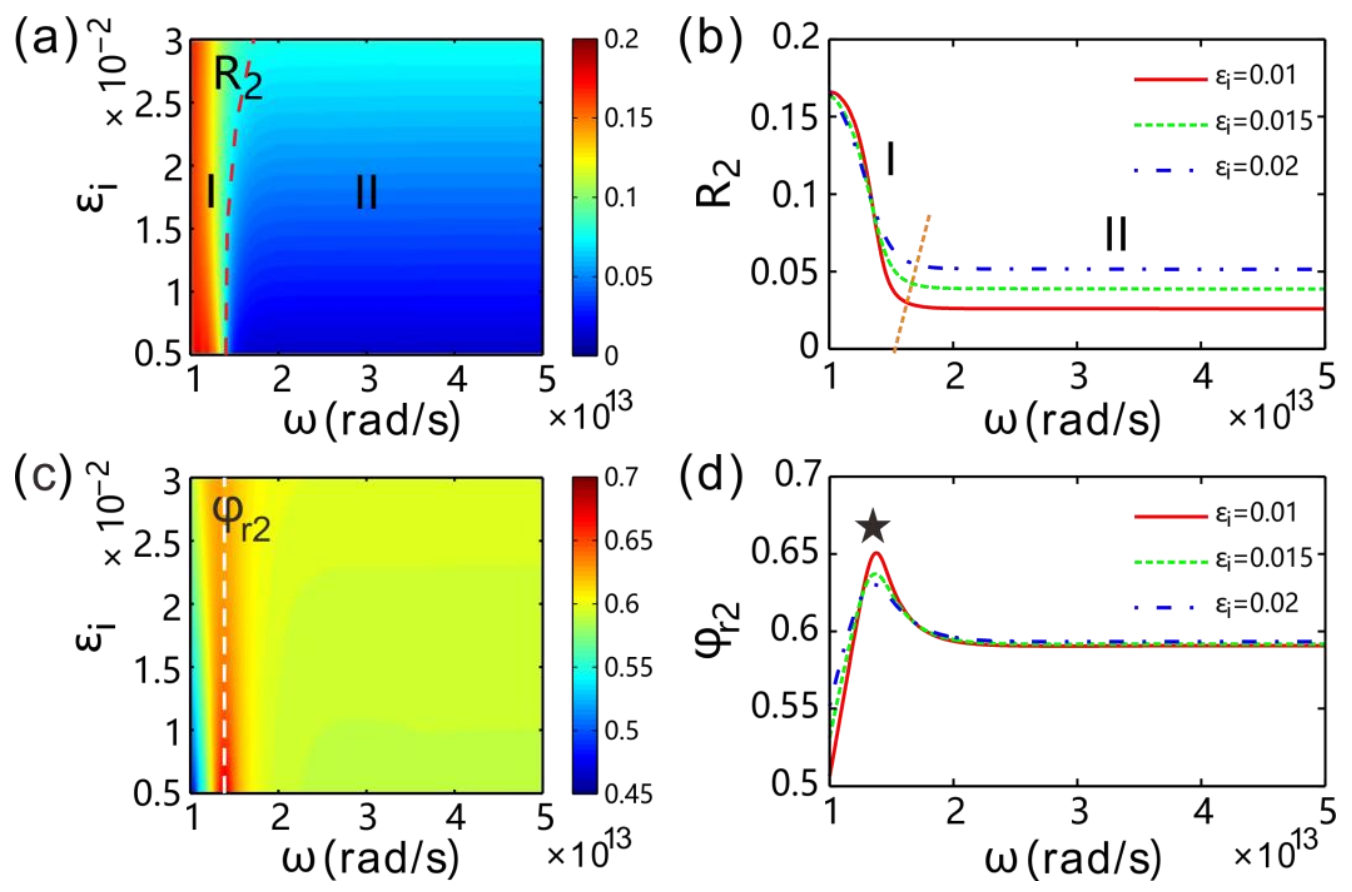

Figure 5. (a,c) The reflectance and phase of reflection coefficient varying with the permittivity and angular frequency for light incident from the right, respectively. (b,d) The reflectance and phase of reflection coefficient varying with the angular frequency for three different permittivities $\varepsilon_{i}=0.01$, 0.015 and 0.02 , respectively.

Figure $5 c$ gives the phase of reflection coefficient in parameter space. We could see the phase varied with the permittivity and angular frequency continuously. For each permittivity, there was a maximum in the phase curve as the angular frequency changed. The maxima of phase located at the position along with the white dotted line in the parameter space. Figure $6 \mathrm{~d}$ illuminates the phase of reflection coefficient varied with the angular frequency for the three fixed permittivities. There is a maximum marked by an asterisk in each curve of phase and the corresponding maximum phase was larger for a smaller permittivity. With the increase of angular frequency, the phase tended to be a constant.

\section{Lateral Shift of Reflected Beam}

As an incident beam impinged upon the structure, the lateral shift of the reflected beam relative to the position predicted by geometry optics could be derived by $\Delta=-d \varphi / d k$ [45]. For several different permittivities $\varepsilon_{i}<\varepsilon_{i 1}=0.02$, Figure 6a plots the lateral shift versus the angular frequency. The shift was positive and there was a peak in each curve around $\omega_{1}$. The maximum shift was larger as the permittivity approached to $\varepsilon_{i 1}$ more. On the other hand, the shift was negative and there was a valley in each curve around $\omega_{1}$ for the permittivities $\varepsilon_{i} \geq \varepsilon_{i 1}$ as shown in Figure 6b. Analogously, the maximum negative shift was larger as the permittivity was more near $\varepsilon_{i 1}$. In the parameter space, Figure $6 \mathrm{c}$ demonstrates the distribution of lateral shift of reflected beam around the $\mathrm{ULP}_{1}$. For better contrast, we took the logarithm of the absolute lateral shift (i.e., $\log _{10}|\Delta / \lambda|$ ). The focusing region was separated into two parts labeled by I and II, respectively. The lateral shift was positive in part I, while it was negative in part II. Except for the ULP 1 , the lateral shift approximated to zero along the dotted line. The negative and positive polarities of lateral shift converted at the $\mathrm{ULP}_{1}$, approving the singularity of $\mathrm{ULP}_{1}$ for the lateral shift of reflected beam.

As light was incident on the lattices from the left, Figure $6 \mathrm{~d}$ gives all of the maxima and minima of lateral shift in each curve. It shows that the positive and negative lateral shift were divided by $\varepsilon_{i 1}$ as the angular frequency changed. In the vicinity of the $\mathrm{ULP}_{1}$, maximum lateral shift dramatically 
climbed when the permittivity increased. The shift tended to infinity as $\varepsilon_{i}$ reached to $\varepsilon_{i 1}$. At the ULP the maxima experienced a step change from positive to negative. And then, the negative shift began to increase sharply with the increase of the permittivity. In our calculating accuracy, the positive and negative lateral shifts reached highly to $4.39 \times 10^{2}$ and $-6.3 \times 10^{2}$ times of the incident wavelength near the $\mathrm{ULP}_{1}$, respectively. Subsequently, the shifts returned to an ordinary magnitude from the negative maximum as the permittivity moved away from the $\mathrm{ULP}_{1}$. On the whole, one can see that the ULP 1 was a singular point of lateral shift and the polarities of lateral shift transformed at this point. Otherwise, around the permittivity $\varepsilon_{i 2}$, the curve of maxima was not smooth enough, indicated by the dashed box. Such an abnormal phenomenon resulted from the parameters close to the $\mathrm{ULP}_{2}$. Distribution of electric field for the boundary mode induced great variation in the phase of reflection coefficient.
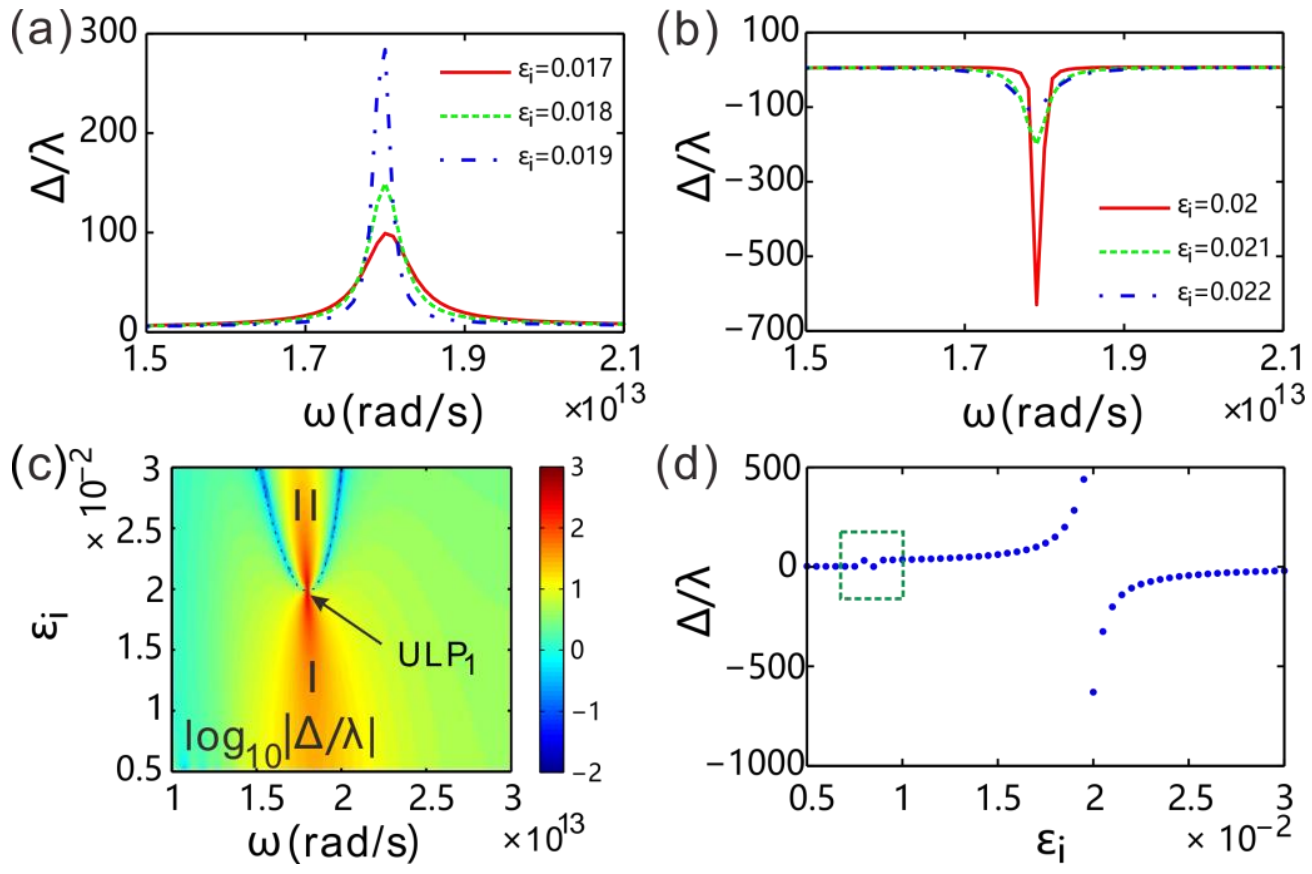

Figure 6. $(\mathbf{a}, \mathbf{b})$ The lateral shift of reflected light versus the angular frequency for several permittivities around $\mathrm{ULP}_{1}$. The permittivity $\varepsilon_{i}<\varepsilon_{i 1}$ for (a) and $\varepsilon_{i}>\varepsilon_{i 1}$ for $(\mathbf{b})$. (c) The lateral shift of reflected light in the vicinity of $\mathrm{ULP}_{1}$. The lateral shift was positive in part I, while it was negative in part II. The results were rescaled by taking logarithm $\log _{10}|\Delta / \lambda|$ for clarity. (d) The maximum lateral shift of reflected light varying with the permittivity around $\mathrm{ULP}_{1}$.

\section{Conclusions}

We studied the reflectance and the phase of the reflection coefficient in complex anti-PT symmetric periodic lattices. The reflection was anisotropic for light incident from the left and right. Two ULPs arose in the parameter space composed of the permittivity and angular frequency. The reflection from the left was greatly enhanced at the ULPs, while the right reflection was suppressed tremendously. Moreover, the phase of the reflected coefficient dislocated around the ULPs. Large lateral shift of reflected beam could be achieved. The maxima of positive and negative lateral shift approached the magnitude of $10^{2} \lambda$ around the ULPs. This study paves the way for the development of unidirectional invisibility, unidirectional lasers and highly sensitive sensors.

Author Contributions: Software, Y.H., F.L., D.Z. (Dong Zhong) and H.X.; formal analysis, D.Z. (Dong Zhao); investigation, H.C. and D.Z. (Dong Zhao); writing-original draft preparation, H.C.; writing — review and editing, H.C., M.F., Y.H, F.L., D.Z. (Dong Zhong) and H.X.; funding acquisition, M.F., H.G., Y.H., F.L. and D.Z. (Dong Zhong).

Funding: National Natural Science Foundation of China (NSFC) (61575148); Natural Science Foundation of Hubei Province (2015CFA040, 2016CFB515); Educational Commission of Hubei Province (D20172803); Scientific Research 
Project of Hubei University of Science and Technology (BK201820, HKCXTD-001, 2016-18X052, KY14048); Colleges and Universities of the National Innovation and Entrepreneurship Training Plan (S201910927024).

Conflicts of Interest: The authors declare no conflicts of interest.

\section{References}

1. Mostafazadeh, A. Non-Hermitian Hamiltonians with a real spectrum and their physical applications. Pramana 2009, 73, 269-277. [CrossRef]

2. Xu, S.L.; Petrovi, N.; Belić, M.R.; Deng, W. Exact solutions for the quintic nonlinear Schrödinger equation with time and space. Nonlinear Dyn. 2017, 84, 251-259. [CrossRef]

3. Xu, S.L.; Petrović, N.; Belić, M.R. Exact solutions of the (2+1)-dimensional quintic nonlinear Schrödinger equation with variable coefficients. Nonlinear Dyn. 2015, 80, 583-589. [CrossRef]

4. Li, Z.P.; Xu, Z.M.; Qu, X.P.; Wang, S.B.; Peng, J.; Mei, L.H. Fabrication of nanopore and nanoparticle arrays with high aspect ratio AAO masks. Nanotechnology 2017, 28, 095301. [CrossRef] [PubMed]

5. Bender, C.M.; Boettcher, S. Real spectra in non-Hermitian Hamiltonians having PT symmetry. Phys. Rev. Lett. 1998, 80, 5243-5246. [CrossRef]

6. Bender, C.M.; Brody, D.C.; Jones, H.F. Complex extension of quantum mechanics. Phys. Rev. Lett. 2002, 89, 270401. [CrossRef]

7. Hatano, N.; Nelson, D.R. Localization transitions in non-Hermitian quantum mechanics. Phys. Rev. Lett. 1996, 77, 570-573. [CrossRef]

8. Ni, H.; Li, M.; Hu, Y.; Mao, C.; Xue, L.; Zeng, H.; Yan, Z.; Wu, Y.; Zheng, C. Two-dimensional SnSe/GeSe van der Waals heterostructure with strain-tunable electronic and optical properties. J. Phys. Chem. Solids 2019, 131, 223-229. [CrossRef]

9. Hu, Y.; Zhang, S.; Xie, M.; Zeng, H. GeSe monolayer semiconductor with tunable direct band gap and small carrier. Appl. Phys. Lett. 2015, 107, 122107. [CrossRef]

10. Xu, S.L.; Xue, L.; Belić, M.R.; He, J.R. Spatiotemporal soliton clusters in strongly nonlocal media with variable potential coefficients. Nonlinear Dyn. 2017, 87, 827-834. [CrossRef]

11. Ke, S.; Liu, Q.; Zhao, D.; Liu, W. Spectral discrete diffraction with non-Hermitian coupling. J. Opt. Soc. Am. B 2018, 35, 2387-2393. [CrossRef]

12. Ke, S.; Liu, J.; Liu, Q.; Zhao, D.; Liu, W. Strong absorption near exceptional points in plasmonic wave guide arrays. Opt. Quantum Electron. 2018, 50, 318. [CrossRef]

13. Li, Z.; Xu, Z.; Qu, X.; Wang, S.; Peng, J. Pattern transfer of hexagonal packed structure via ultrathin metal nanomesh masks for formation of Si nanopore arrays. J. Alloys Compd. 2017, 695, 458-461. [CrossRef]

14. Li, Z.; Wang, G.; Li, Z.; Cheng, Z.; Zhou, G.; Li, S. Flexible transparent electrodes based on gold nanomeshes. Nanoscale Res. Lett. 2019, 14, 132. [CrossRef] [PubMed]

15. Zhao, D.; Zhong, D.; Hu, Y.; Ke, S.; Liu, W. Imaginary modulation inducing giant spatial Goos-Hänchen shifts in one-dimensional defective photonic lattices. Opt. Quantum Electron. 2019, 51, 113. [CrossRef]

16. Zhao, D.; Ke, S.; Liu, Q.; Wang, B.; Lu, P. Giant Goos-Hänchen shifts in non-Hermitian dielectric multilayers incorporated with graphene. Opt. Express 2018, 26, 2817-2828. [CrossRef] [PubMed]

17. Xu, S.L.; Zhao, Y.; Petrović, N.Z.; Belić, M.R. Spatiotemporal soliton supported by parity-time symmetric potential with competing nonlinearities. EPL 2016, 115, 14006. [CrossRef]

18. Xu, S.L.; Petrović, N.; Belić, M.R.; Hu, Z.L. Light bullet supported by parity-time symmetric potential with power-law nonlinearity. Nonlinear Dyn. 2016, 84, 1877-1882. [CrossRef]

19. Wang, H.; Kong, W.; Zhang, P.; Li, Z.; Zhong, D. Coherent perfect absorption laser points in one-dimensional anti-parity-time-symmetric photonic crystals. Appl. Sci. 2019, 9, 2738. [CrossRef]

20. Makris, K.G.; El-Ganainy, R.; Christodoulides, D.N. Beam dynamics in PT symmetric optical lattices. Phys. Rev. Lett. 2008, 100, 103904. [CrossRef]

21. Ke, S.; Zhao, D.; Liu, Q.; Liu, W. Adiabatic transfer of surface plasmons in non-Hermitian graphene waveguides. Opt. Quantum Electron. 2018, 50, 393. [CrossRef]

22. Longhi, S. Quantum-optical analogies using photonic structures. Laser Photonics Rev. 2009, 3, $243-261$. [CrossRef]

23. Chen, S.; Guo, Q.; Xu, S.; Belic, M.R.; Zhao, Y.; Zhao, D.; He, J. Vortex Solitons in Bose-Einstein Condensates with Spin-Orbit Coupling and Gaussian Optical Lattices. Appl. Math. Lett. 2019, 92, 15-21. [CrossRef] 
24. Ke, S.; Zhao, D.; Liu, Q.; Wu, S.; Wang, B.; Lu, P. Optical imaginary directional couplers. J. Lightwave Technol. 2018, 36, 2510-2515. [CrossRef]

25. Feng, L.; Zhu, X.; Yang, S.; Zhu, H.; Zhang, P.; Yin, X.; Wang, Y.; Zhang, X. Demonstration of a large-scale optical exceptional point structure. Opt. Express 2014, 22, 1760-1767. [CrossRef] [PubMed]

26. Zhu, X.F. Defect states and exceptional point splitting in the band gaps of one-dimensional parity-time lattices. Opt. Express 2015, 23, 22274-22284. [CrossRef] [PubMed]

27. Zhao, D.; Wang, Z.Q.; Long, H.; Wang, K.; Wang, B.; Lu, P.X. Optical bistability in defective photonic multilayers doped by graphene. Opt. Quantum Electron. 2017, 49, 163. [CrossRef]

28. Meng, P.; Zhao, D.; Zhong, D.; Liu, W. Topological plasmonic modes in graphene-coated nanowire arrays. Opt. Quantum Electron. 2019, 51, 156. [CrossRef]

29. Li, Z.; Xu, Z.; Li, D.; Wu, A.; Ruan, R. A nanoporous GaN photoelectrode on patterned sapphire substrates for high-efficiency photoelectrochemical water splitting. J. Alloys Compd. 2019, 803, 748-756. [CrossRef]

30. Li, Z.; Li, D.; Wu, A.; Ruan, R.; Xu, Z. Fabrication of GaN truncated nanocone array using a pre-deposited metallic nano-hemispheres template for efficient solar water splitting. Nanotechnology 2019, 30, 405302. [CrossRef]

31. Ke, S.; Wang, B.; Qin, C.; Long, H.; Wang, K.; Lu, P. Exceptional points and asymmetric mode switching in plasmonic waveguides. J. Lightwave Technol. 2016, 34, 5258-5262. [CrossRef]

32. Lin, Z.; Ramezani, H.; Eichelkraut, T.; Kottos, T.; Cao, H.; Christodoulides, D. Unidirectional invisibility induced by PT-symmetric periodic structures. Phys. Rev. Lett. 2011, 106, 213901. [CrossRef] [PubMed]

33. Yang, F.; Liu, Y.; You, L. Anti-PT symmetry in dissipatively coupled optical systems. Phys. Rev. A 2017, 96, 053845. [CrossRef]

34. Ke, S.; Zhao, D.; Liu, J.; Liu, Q.; Liao, Q.; Wang, B.; Lu, P. Topological bound modes in anti-PT-symmetric optical waveguide arrays. Opt. Express 2019, 27, 13858-13870. [CrossRef] [PubMed]

35. Zhang, X.; Jiang, T.; Sun, H.; Chan, C.T. Dynamically encircling an exceptional point in anti-PT-symmetric systems: Asymmetric mode switching for symmetry-broken states. arXiv, 2018; arXiv:1806.07649.

36. Zhao, D.; Liu, W.W.; Ke, S.L.; Liu, Q.J. Large lateral shift in complex dielectric multilayers with nearly parity-time symmetry. Opt. Quantum Electron. 2018, 50, 323. [CrossRef]

37. Fang, Y.; Ouyang, Z. An approximately omnidirectional defect mode of the TE wave from one-dimensional photonic crystals doped by negative-index materials. J. Opt. A Pure Appl. Opt. 2009, 11, 045103. [CrossRef]

38. Zhao, D.; Ke, S.; Hu, Y.; Wang, B.; Lu, P. Optical bistability in parity-time-symmetric dielectric multilayers incorporated with graphene. J. Opt. Soc. Am. B 2019, 36, 1731-1737. [CrossRef]

39. Rüter, C.E.; Makris, K.G.; El-Ganainy, R.; Christodoulides, D.N.; Segev, M.; Kip, D. Observation of parity-time symmetry in optics. Nat. Phys. 2010, 6, 192-195. [CrossRef]

40. Feng, L.; Xu, Y.L.; Fegadolli, W.S.; Lu, M.; Oliveira, J.E.B.; Almeida, V.R.; Chen, Y.; Scherer, A. Experimental demonstration of a unidirectional refectionless parity-time metamaterial at optical frequencies. Nat. Mater. 2013, 12, 108-113. [CrossRef] [PubMed]

41. Regensburger, A.; Bersch, C.; Miri, M.A.; Onishchukov, G.; Christodoulides, D.N.; Peschel, U. Parity-time synthetic photonic lattices. Nature 2012, 488, 167-171. [CrossRef] [PubMed]

42. Merano, M. Optical beam shifts in graphene and single-layer boron-nitride. Opt. Lett. 2016, 41, 5780-5783. [CrossRef] [PubMed]

43. Zhao, D.; Liu, F.; Meng, P.; Wen, J.; Xu, S.; Li, Z.; Zhong, D. Reflection enhancement and giant lateral shift in defective photonic crystals with graphene. Appl. Sci. 2019, 9, 2141. [CrossRef]

44. Longhi, S.; Della Valle, G.; Staliunas, K. Goos-Hänchen shift in complex crystals. Phys. Rev. A 2011, 84, 042119. [CrossRef]

45. Ma, P.; Gao, L. Large and tunable lateral shifts in one-dimensional PT-symmetric layered structures. Opt. Express 2017, 25, 9676-9688. [CrossRef] [PubMed]

(C) 2019 by the authors. Licensee MDPI, Basel, Switzerland. This article is an open access article distributed under the terms and conditions of the Creative Commons Attribution (CC BY) license (http://creativecommons.org/licenses/by/4.0/). 